\title{
Problems related to cytomegalovirus infection and biliary atresia
}

\author{
C Zabiegaj-Zwick, E Nel, S W Moore
}

Background. Human cytomegalovirus (CMV) infection is related to biliary disease, being cholestatic in its own right. It has also been associated with intrahepatic bile duct destruction and duct paucity, indicating a possible role in the pathogenesis and progression of extrahepatic biliary atresia (BA). BA patients who are CMVIgM-positive appear to have greater liver damage than uninfected patients, consequently affecting outcome.

Methods. We reviewed the medical records of 74 patients diagnosed with hepatobiliary disease between 2000 and 2011, assessing clinical outcome and potential risk factors. Patients, categorised into those with and those without BA, were compared in terms of CMV infection.

Results. The 74 patients included 39 (52\%) BA and 35 non-BA patients; following the exclusion of patients due to insufficient data,
27 (69\%) BA and 31 non-BA patients were reviewed. Twenty-one (78\%) BA patients were CMV-positive (IgM/IgG), including 20 IgM-positive patients versus 8 in the non-BA group $(p<0.01)$. Two (7.5\%) CMV-IgM-positive BA infants were HIV-exposed versus 7 $(35 \%)$ in the non-BA group $(p<0.01)$. Long-term outcomes included 3 deaths in the non-HIV CMV-positive group and a higher rate of severe early liver damage, suggesting a poorer outcome in CMVaffected patients.

Conclusions. Our results suggest a correlation between CMV exposure/infection and BA which affects clinical outcome. HIVpositivity does not preclude BA and should be investigated further.

S Afr Med J 2012;102(11):890-892. DOI:10.7196/SAMJ.6163
Biliary atresia (BA) is a neonatal cholestatic jaundice which results from obstruction of the extrahepatic biliary system due to destructive inflammation of unknown aetiology. It occurs in 1/5 - 1/8 000 live births in Asia and 1/18 - 1/20 000 children in Europe. ${ }^{1,2}$ Apart from this difference in incidence, no ethnic differences have been demonstrated. However, females appear to carry a slightly higher BA risk. BA affects variable lengths of the extrahepatic system and, if untreated, most patients die of biliary cirrhosis before their second birthday due to liver failure. Recognised subtypes include an embryonic form (BA splenic malformation (BASM)) associated with other congenital anomalies, as well as at least 3 other subtypes (isolated BA, a cystic variety, and cytomegalovirus (CMV)IgM-positive BA). If treated timeously by surgical drainage (Kasai procedure), approximately $40-55 \%$ of affected children can clear their jaundice to normal values and expect at least a 5-year native liver survival. ${ }^{2}$ BA is the leading cause of end-stage liver disease in children, leading to significant mortality and morbidity; ${ }^{3}$ therefore, research into potential causes would improve results, and possibly prevention.

The aetiology of BA is unknown, but it is generally agreed to be attributed to multifactorial prenatal and perinatal insults to the developing biliary tree. Research points towards a viral-initiating factor in predisposed individuals, which initiates an antigen-antibody reaction that causes inflammatory targeting of the extrahepatic (and possibly intrahepatic) bile ducts. ${ }^{4}$ This mechanism is of particular interest in patients with impaired immunity (e.g. HIV-infected).

Division of Paediatric Surgery, Stellenbosch University, Tygerberg, Cape Town C Zabiegaj-Zwick, MB ChB

S W Moore, MB ChB, FRCS (ED), MD

Division of Paediatrics, Stellenbosch University, Tygerberg, Cape Town E Nel, MB ChB, MMed, BSc (Hons) (Stell)

Corresponding author: S W Moore (swm@sun.ac.za)
CMV is related to biliary disease in infants, being cholestatic in its own right, ${ }^{5}$ and has been implicated in intrahepatic bile destruction and duct paucity, as a potential causative factor. ${ }^{6,7}$ Patients with IgMCMV-positivity appear to suffer progressive liver damage, suggesting that the virus may promote ongoing sclerosis in the biliary tree, consequently affecting outcome. ${ }^{8}$

We aimed: (i) to detect CMV infection rates and baseline characteristics of infants with BA over 10 years (2001 - 2011); and (ii) to investigate the effect of perinatal CMV infection in BA and compare the outcomes of CMV-IgM-positive children with nonexposed children with cholestatic jaundice.

\section{Methods}

We performed a retrospective review of hospital records of patients with cholestatic jaundice, referred to the Paediatric Surgical Unit of Tygerberg Hospital from 2001 to 2011 (Table 1). Data were anonymously collated and stored in a Microsoft Excel database. Patients were categorised into 2 groups: those with and those without BA (non-BA), and were compared in terms of CMV infection. CMV testing results were derived from laboratory results or patient records. Fisher's exact and/or chi-squared statistical tests for significance were performed where

\begin{tabular}{ll}
\hline \multicolumn{2}{l}{ Table 1. Causes of cholestatic jaundice in a cohort of 74 patients } \\
\hline Cause & $N$ \\
\hline Biliary atresia & $39(27$ evaluable $)$ \\
Non-biliary atresia & $34(31$ evaluable $)$ \\
Neonatal hepatitis & 10 \\
Choledochal cyst & 9 \\
Viral enterocolitis & 5 \\
HIV hepatitis & 3 \\
Autlstones & 1 \\
Miscellaneous & 1
\end{tabular}


applicable. A $p$-value $<0.05$ was regarded as statistically significant. Ethical approval of the study was granted (S12/02/061) by the ethics review committee of Stellenbosch University. Research was conducted according to guidelines outlined in the Declaration of Helsinki. Patient anonymity and confidentiality were protected.

\section{Results}

Of the 74 patients investigated, 39 (52\%) had BA and 35 had other causes of surgical hepatobiliary disease (Table 1); 27 (69\%) BA patients and $31(89 \%)$ non-BA patients were reviewed following the exclusion of $12 \mathrm{BA}$ patients and 4 non-BA patients due to lack of sufficient data. Twenty-one (78\%) BA patients had CMV positivity (IgM/IgG) on testing; 20 were IgM-positive, whereas 8 non-BA cholestatic jaundiced patients were IgM-positive $(p<0.01)$. Two (7.5\%) of $27 \mathrm{BA}$ infants were HIV-exposed (born to HIV-positive mothers), whereas 7 (35\%) of the non-BA group were HIV-positive $(p<0.01)$. Both HIV-exposed BA infants were CMV-IgM-positive. Long-term outcomes of the $21 \mathrm{CMV}$-positive BA patients (non-HIV exposed) included 3 deaths and a higher rate of severe early liver damage, suggesting a poorer outcome in CMV-affected patients.

\section{Discussion}

The surgical causes of prolonged neonatal jaundice include BA, hypoplasia of bile ducts, inspissated bile ducts, choledochal cysts and spontaneous bile duct perforation. BA remains the most common neonatal cholestatic disorder and is characterised histologically by complete obliteration of the lumen of all, or part, of the extrahepatic biliary tree.

Although the cause of BA is unknown and many theories as to its aetiology exist, it is generally agreed that it is attributed to multifactorial pre- and postnatal insults to the developing biliary tree. . $, 7,10,11$ Current aetiology theories include viral infections, including human CMV; immune dysregulation; auto-immune mechanisms; vascular lesions; defective morphogenesis (including inherited mutations of laterality genes, somatic mutations, and modifier genes); and toxin exposure. ${ }^{4} \mathrm{~A}$ study showing a seasonal clustering of children presenting with BA supports an infective aetiological factor. ${ }^{12}$ Although multiple factors are involved in the targeting of the developing biliary tree, the correlation between viral infections and BA suggests the possibility that the viral infection initiates an inflammatory auto-immune process which results in the ductal sclerosis seen in the condition. ${ }^{13}$ Current information suggests that this is based on antibody-mediated inflammation (probably virally induced) which leads to progressive biliary sclerosis. ${ }^{4}$

Histological observations in the porta hepatis and liver samples of children with BA provide further support of a possible link between BA and a viral infection. ${ }^{14}$ Viruses currently identified as potential aetiological agents include CMV, reovirus, and herpes simplex virus, among others. ${ }^{15}$ Although there appears to be a correlation between CMV infection and BA, the significance must still be determined due to the small cohorts studied thus far., ${ }^{4,5,7,9-11,16,17}$

Human CMV infection is a strong candidate, being cholestatic in its own right. ${ }^{5,9}$ It has also been associated with intrahepatic bile duct destruction and ductal paucity, indicating a possible role in the pathogenesis and progression of extrahepatic BA. ${ }^{7,8}$ In addition, when related to BA, CMV-IgM-positive patients appear to have greater liver damage, consequently affecting outcome deleteriously., ${ }^{8,10,18}$

Although no unequivocal link has been established between BA and CMV infection, there appears to be a fairly strong correlation between the two, as demonstrated by our study. CMV is a slow replicating virus from the herpes family, infecting only as many as $1 \%$ of all neonates in developed countries, ${ }^{9}$ but demonstrating up to $90 \%$ IgG-positivity in developing countries. ${ }^{19}$ Only $5-10 \%$ of infected infants have typical CMV inclusion disease with symptoms of hepatosplenomegaly, jaundice and petechiae; a further $5 \%$ having subclinical disease. Consequently, the vast majority of exposed babies are asymptomatic. The South African population has a high background CMV infection rate in pregnant women (M Anderson, personal communication); in this context, it is therefore difficult to determine when CMV infection is the unequivocal cause of BA. Nevertheless, there appears to be a connection to BA in countries with low CMV community prevalence. ${ }^{2}$

A principal difficulty in making the connection between these entities is that no single test is $100 \%$ sensitive and/or specific for CMV infection..$^{1,9,13,16}$ Serology, CMV quantitative polymerase chain reaction (qPCR), immunohistochemistry and histology have been unable to confirm a possible role of CMV in BA aetiology. De Tomasso et al. ${ }^{9}$ describe a low accuracy of serological tests for detecting active CMV infection, with no correlation between the CMV-positive qPCR and histopathological changes reported by others. ${ }^{1,13,16}$ Our study, however, reinforces a probable correlation.

Some information suggests a worse outcome in CMV-affected patients. ${ }^{8}$ Hill et al. ${ }^{14}$ have shown a connection to CMV Th1 and Th17 cell infiltrates which affects BA prognosis. This raises the possibility that CMV infection might not only be a causative factor, but may promote ongoing biliary tree sclerosis. ${ }^{10,18}$

The presence of co-morbidities such as HIV raises interesting possibilities in the antigen-antibody hypothesis of BA aetiology. This is of particular interest in areas of high HIV prevalence, such as sub-Saharan Africa, and is probably explained by the fact that some non-BA patients with neonatal hepatitis on biopsy were subsequently shown to be HIV-related. Our results, however, demonstrate that HIV exposure did not preclude the occurrence of BA in at least 2 cases. Recent evidence indicates that an interaction between HIV infection and other environmental factors such as CMV co-infection accelerates immune system deterioration..$^{20}$ On the other hand, should an antigen-antibody be important in the aetiology of BA, impaired immunity, such as HIV, may influence BA type and/or outcome. As such, this connection warrants further study.

\section{Conclusion}

Our results suggest a correlation between CMV exposure, infection and surgical hepatobiliary disease, including an effect on BA outcome. HIV positivity does not preclude BA; a relationship that requires further investigation.

\section{References}

1. Jevon GP, Dimmick JE. Biliary atresia and cytomegalovirus: A DNA study. Pediatr Dev Patho 1999;2:11-14. [http://dx.doi.org/10.1007/s100249900083]

2. Davenport M. Biliary atresia: Clinical aspects. Semin Pediatr Surg 2012:21(3):175-184. [http://dx.do. org/10.1053/j.sempedsurg.2012.05.010]

3. Schreiber RA, Barker CC, Roberts EA, Martin SR. Biliary atresia in Canada: The effect of centre caseload experience on outcome. J Paediatr Gastroenterol Nutr 2010;51(1):61-65. [http://dx.doi. org/10.1097/MPG.0b013e3181d67e5e]

4. Sokol RJ, Mack C, Narkewicz MR, Karrer FM. Pathogenesis and outcome of biliary atresia: curren concepts. J Paediatr Gastroenterol Nutr 2003;27(3):4-21. [http://dx.doi.org/10.1097/00005176

5. Oliveira NL, Kanawaty FR, Costa SC, Hessel G. Infection by cytomegalovirus in patients with neonatal cholestasis. Arq Gastroenterol 2002;39(2):132-136.

6. Fischler B, Ehrnst A, Forggren M, et al. The viral association of neonatal cholestasis in Sweden: a possible link between cytomegalovirus infection and extrahepatic biliary atresia. J Pediat Gastroenterol Nutr 1998;27(1):57-64. [http://dx.doi.org/10.1097/00005176-199807000-00010]

7. Mohanty S, Shah I, Bhatnagar S. Evolving biliary atresia with cytomegalovirus. Indian Pediat 2011;48(8):644-646

8. Fischler B, Svensson JF, Nemeth A. Early cytomegalovirus infection and the long-term outcome of biliary atresia. Acta Paediatr 2009;98(10):1600-1602. [http://dx.doi.org/10.1111/j.1651 2227.2009.01416.x]

9. De Tommaso A, Andrade PD, Costa SC, et al. High frequency of human cytomegalovirus DNA in the liver of infants with extrahepatic neonatal cholestasis. BMC Infect Dis 2005;5:108-115. [http://dx.do org/10.1186/1471-2334-5-108

10. Shen C, Zheng S, Wang W, Xiao X-M. Relationship between prognosis of biliary atresia and infection of cytomegalovirus. World J of Pediatr 2008;(2):123-126. [http://dx.doi.org/10.1007/s12519-0080024-8]

1. Amer OT, Abd El-Rahma HA, Sherief LM, et al. Role of some viral infections in neonatal cholestasis. Egypt I Immunol 2004;1(2):149-155. 


\section{RESEARCH}

12. Livesey E, Cortina Borja M, Sharif K, et al. Epidemiology of biliary atresia in England and Wales 1999 - 2006). Arch Dis Child Fetal Neonatal Ed 2009;94(6):F451-F455.

13. Pawlowska J, Swiatkowska E, Gliwicz D, et al. The role of cytomegalovirus infection in pathogenesis of neonatal cholestasis. E\&C Hepatology 2010;6(1):25-29.

14. Hill R, Hussain M, Quaglia A, et al. TH-17 cells infiltrate the liver in biliary atresia and are related to prognosis. Proceedings of the 3rd EUPSA/BAPS Combined Congress, Rome, Italy, 13 - 16 June

15. Soomro GB, Abbas Z, Hassan M, et al. Is there any association of extra hepatic biliary atresia with cytomegalovirus or other infections? J Pak.Med Assoc 2011;61(3):281-283.

16. Bellomo-Brandao MA, Andrade PD, Costa SC, et al. Cytomegalovirus frequency in neonatal intrahepatic cholestasis determined by serology, histology, immunohistochemistry and PCR. World J Gastroenterol 2009;15(27):3411-3416. [http://dx.doi.org/10.3748/wgg.15.3411]
17. Tarr PI, Haas JE, Christie DL. Biliary atresia, cytomegalovirus and age at referral Pediatrics

1996;97(6):828-831.
18. Spearman CW, McCulloch M, Millar AJ, Burger H. Liver transplantation at Red Cross War Memorial Children's Hospital. S Afr Med J 2006;96(9):960-963.

19. Cannon MJ, Schmid DS, Hyde TB. Review of cytomegalovirus seroprevalence and demographi characteristics associated with infection. Rev Med Virol 2010;20(4):202-213. [http://dx.doi

20. Deeks SG, Verdin E, McCune JM. Immunosenescence and HIV. Curr Opin Immunol 2012 (in press).

Accepted 10 September 2012 